\title{
Social Competence at the Playground: Preschoolers During Recess
}

\author{
Guida Veiga ${ }^{a, b, *}$, Wendy de Leng ${ }^{c}$, Ricardo Cachucho ${ }^{d}$, \\ Lizet Ketelaar, ${ }^{c}$, Joost N. Kok ${ }^{d}$, Arno Knobbe ${ }^{d}$, \\ Carlos Neto ${ }^{f}$ and Carolien Rieffe ${ }^{\mathrm{c}, \mathrm{e}}$ \\ ${ }^{a}$ Departamento de Desporto e Saúde, Escola de Ciências e Tecnologia, Universidade \\ de Évora, Évora, Portugal \\ ${ }^{\mathrm{b}}$ Laboratory of Motor Behavior, Faculdade de Motricidade Humana, Universidade \\ de Lisboa, Lisboa, Portugal \\ ${ }^{\mathrm{c} D e v e l o p m e n t a l}$ Psychology, Leiden University, Leiden, The Netherlands \\ ${ }^{\mathrm{d}}$ Leiden Institute for Advanced Computer Science, Leiden University, Leiden, The \\ Netherlands \\ ${ }^{\mathrm{e}}$ Dutch Foundation for the Deaf and Hard of Hearing Child, Amsterdam, The \\ Netherlands \\ ${ }_{\mathrm{f}}^{\mathrm{L}}$ aboratory of Motor Behavior, CIPER, Faculdade de Motricidade Humana, \\ Universidade de Lisboa, Lisboa, Portugal
}

Social interactions at the playground have been represented as a rich learning opportunity to hone and master social skills at preschool years. Specifically, all forms of social play (fantasy, role, exercise or rough-and-tumble) have been related to children's social competence. The main goal of this study was to examine whether it is a certain kind of social play which facilitates the development of social competence, or if it is just the opportunity for interacting during recess that provides children with an optimal environment for social learning. A total of 73 preschoolers (4-6 years old) were videotaped at the school's playground. Teachers provided assessments of children's social competence. Children's interactions at the playground were assessed through an innovative measuring method, based on radio-frequency identification devices. The results showed a positive association between exercise play and children's social competence. In contrast with the literature, both forms of pretend play, fantasy and role play were unrelated to children's social competence. Smaller peer groups and longer interactions also demonstrated a positive association with these preschoolers' social competence. The study shows the importance

*Correspondence to: Guida Veiga, Departamento de Desporto e Saúde, Escola de Ciências e Tecnologia, Universidade de Évora, Portugal. E-mail: gveiga@uevora.pt 